\title{
Interval timing and time-based decision making
}

\author{
Warren H. Meck' ${ }^{1}$ Valérie Doyère ${ }^{2}$ and Agnès Gruart ${ }^{3 *}$ \\ 1 Duke University, Durham, NC, USA \\ 2 CNRS, Université Paris-Sud, Orsay, France \\ ${ }^{3}$ University Pablo de Olavide, Seville, Spain \\ *Correspondence: agrumas@upo.es
}

The importance of time perception and timed performance is revealed in everyday activities from the sleep-wake cycle to verbal communication, playing, and appreciating music, the exquisite temporal control of both voluntary and involuntary behavior, and choice. With regard to the last point, making decisions is heavily influenced by the duration of the various options, the duration of the expected delays for receiving the options, and the time constraints for making a choice. Recent advances suggest that the brain represents time in a distributed manner and reflects time as a result of temporal changes in network states and/or by the coincidence detection of the phase of different neural populations. Moreover, the oscillatory properties of neural circuits can be shown to influence the acquisition of conditioned responding and the timing of motor responses. This Research Topic on "Interval Timing and Time-Based Decision Making" emerged from a symposium sponsored by the European COST-Action on Time In MEntaL activity: theoretical, behavioral, bioimaging, and clinical perspectives (TIMELY) that was a satellite of the European Brain and Behaviour Society meeting held in Seville, Spain (September 9, 2011). The focus of that TIMELY symposium was on "Neurobiology of Time Perception: From Normality to Dysfunction" and was organized by Valérie Doyère, Argiro Vatakis, and Elzbieta Szelag. The current volume contains 58 state-of-the-art original research, opinion, and review articles exploring the relationships between time and decision making with respect to the underlying psychological and biological mechanisms. The overall goal is to examine how time in the physical world is reconstructed, distorted, and modified in brain networks by emotion, learning, and neuropathology.

The contributions to the Research Topic have been divided by the co-editors into nine main categories in order to organize the breadth of coverage, from animal to human research. These categories were selected based upon research strategy and experimental techniques, and include the following:
(1) Perception, representation, and categorization

(2) Emotional distortions

(3) Decision making and learning

(4)Development of timing and counting

(5) Neuroimaging and pathophysiology

(6) Temporal conditioning and neurophysiology

(7)EEG/ERP analyses and the role of the supplementary motor area

(8) Molecular, neuropharmacological, and neuroendocrine mechanisms

(9)Neural-network models

As papers often may belong to more than a single category, we apologize if some authors feel they have been "misplaced," but we tried to maintain equilibrium among the different categories without being unfaithful to the authors' main message.

It has been a great pleasure to be involved in this Research Topic as much progress has been made in exploring the temporal horizons of the mind and brain in the past few years. Much more work needs to be done and many issues remain to be addressed before we fully understand the functional and neural mechanisms of interval timing. We would like to thank all of the authors, reviewers, and Frontiers staff for helping to make this Research Topic possible and we look forward to further explorations of interval timing and time-based decision making.

Received: 14 March 2012; accepted: 14 March 2012; published online: 30 March 2012. Citation: Meck WH, Doyère V and Gruart A (2012) Interval timing and time-based decision making. Front. Integr. Neurosci. 6:13. doi: 10.3389/fnint.2012.00013

Copyright (c) 2012 Meck, Doyère and Gruart. This is an open-access article distributed under the terms of the Creative Commons Attribution Non Commercial License, which permits non-commercial use, distribution, and reproduction in other forums, provided the original authors and source are credited. 